\title{
Frequency, duration and predictors of bronchiolitis episodes of care among infants $\geq 32$ weeks gestation in a large integrated healthcare system: a retrospective cohort study
}

Valerie J Flaherman ${ }^{1 *}$, Arona I Ragins ${ }^{2}$, Sherian Xu Li ${ }^{2}$, Patricia Kipnis ${ }^{2,3}$, Anthony Masaquel ${ }^{4}$ and Gabriel J Escobar ${ }^{2,5}$

\begin{abstract}
Background: Bronchiolitis is common in the first two years of life and is the most frequent cause of hospitalization in this age group. No previous studies have used an episode-of-care analysis to describe the frequency, duration, and predictors of bronchiolitis episodes of care during the first two years.

Methods: We conducted a retrospective cohort study of 123,264 infants $\geq 32$ weeks gestation born at 6 Northern California Kaiser Permanente hospitals between 1996 and 2002. We used electronic medical records to concatenate hospital, emergency department and outpatient health care encounters for bronchiolitis into discrete episodes of care. We used descriptive statistics to report frequency and duration of bronchiolitis episodes and used logistic regression to assess the effect of gestational age and other clinical and demographic predictors on the outcome of bronchiolitis episodes.

Results: Among all infants, the rate of bronchiolitis episodes was 162 per 1000 children during the first 2 years of life; approximately $40 \%$ required $>1$ day of medical attention with a mean duration of $7.0 \pm 5.9$ days. Prematurity was associated with increased risk of bronchiolitis episodes and longer duration. Bronchiolitis episodes rates per 1000 infants were 246 for 32-33 weeks gestational age, 204 for 34-36 weeks, and 148-178 for $>36$ weeks. Male gender, African-American and Hispanic race/ethnicity, and parental history of asthma were associated with an increased risk of having a bronchiolitis episode and/or longer duration.

Conclusions: Bronchiolitis episodes of care are frequent during the first two years of life and the duration ranges from 1 to 27 days. Prematurity was associated with more frequent and longer duration of bronchiolitis episodes of care, which may reflect illness severity and/or perceived vulnerability.
\end{abstract}

\section{Background}

Bronchiolitis is the most common lower respiratory condition in children younger than 2 years [1], and presents with a wide spectrum of clinical illness, from mild symptoms to severe, life-threatening respiratory distress. More severe bronchiolitis is the most frequent cause of infant hospitalization in the United States [2,3] with costs estimated at $\$ 543$ million per year [4]. Bronchiolitis also causes a substantial emergency department (ED) and outpatient burden [5-7]. Populations at

\footnotetext{
* Correspondence: FlahermanV@peds.ucsf.edu

${ }^{1}$ Department of Pediatrics, University of California, 3333 California St, Box 0503, San Francisco, CA 94143-0503, USA

Full list of author information is available at the end of the article
}

increased risk for severe bronchiolitis include racial and ethnic minorities and those who had preterm birth, bronchopulmonary dysplasia (BPD) or congenital heart disease. $[6,8,9]$

Previous research on the epidemiology of bronchiolitis has focused on ascertaining rates of outpatient care, ED visits, and hospitalizations, with widely differing results that can be attributed to differences in study designs, methodologies, and populations. Estimates of the rate of outpatient visits for children $<2$ years range from 103 per 1000 children [2] to 238 visits per 1000 child years [5]. Estimates of the rate of hospitalization also vary. Koehoorn et al. [10] found a hospitalization rate of 17.1 per 1000 child years for children $<1$ year. Shay et al., 
estimated similar rates of hospitalization in the US for infants $<1$ year ranging from 12.9 per 1000 in 1980 to 31.2 per 1000 in 1996 [11], whereas Carroll et al. [5] estimated a higher rate of 71 per 1000 child years during 1995-2003. Carroll et al. [5] also estimated a rate of 77 ED visits per 1000 child years. Respiratory syncytial virus is an important cause of bronchiolits, and recent research has examined the prevalence of respiratory syncytial virus (RSV) among children presenting for medical care, and found that $15-20 \%$ of children presenting for care had RSV infection or co-infection [12].

These existing studies have tabulated the frequency of hospitalizations and individual outpatient visits for bronchiolitis, and have estimated the frequency of RSV infection among those receiving care, but have not provided a comprehensive description of the healthcare utilization for each infant over a specific time period. In order to provide such a comprehensive description, health care effectiveness researchers have promoted "episode of care" analysis. Hornbrook et al. [13] first defined an episode as a "series of temporally contiguous health services related to treatment of a given spell of illness or provided in response to a specific request by the patient." Recently episode analysis has been used as an important tool for estimating costs, determining patterns of use, evaluating outcomes of care, and understanding medical practice variation [13-15], using the entire duration of care as the basis for measurement, with the assumption that this will be more effective than counting single outpatient visits or single hospitalizations $[13,14,16]$. To the best of our knowledge, no previous studies have defined episodes of care for bronchiolitis or have reported their frequency, duration or risk during the first 2 years of life.

In this study, we use an "episodes of care" (henceforth, "episode" or EOC) approach to determine the frequency, duration and risk factors for bronchiolitis in children $<2$ years among members of a large, integrated healthcare system in Northern California. Using this approach, we analyze our results by gestational age (GA) to examine the burden of disease among those born preterm, who are considered at high risk for bronchiolitis [17]. Below we describe the frequency, duration and characteristics of bronchiolitis episodes and the clinical and demographic predictors of bronchiolitis episodes longer than one day among preterm and term infants.

\section{Methods}

\section{Study design}

This study was approved by the Kaiser Permanente Medical Care Program (KPMCP) Institutional Review Board for the Protection of Human Subjects, which has jurisdiction over all the hospitals and clinics described in this study. We conducted a retrospective cohort study that included infants $\geq 32$ weeks GA born and discharged alive from one of 6 Northern California KPMCP hospitals between 1996 and 2004. Infants $<32$ weeks GA at birth represent a large portion of all infants who receive RSV prophylaxis in our integrated healthcare system and represent only a small portion of all preterm infants born annually [18]. Consequently, infants $<32$ weeks GA were not included in this study due to routine administration of RSV immunoprophylaxis. To reduce the effects of loss to follow up on our analysis we identified those infants who remained KPMCP members until they were two years of age. We included subjects in the cohort if they were members for $\geq 20$ of the 24 months after the infant's birth, and if they had no more than two consecutive months without membership. Subjects were defined as members for each month in the study if they were either insured by KPMCP or used KPMCP medical services. See Figure 1 for flowchart of the cohort selection.

We extracted data regarding these infants from KPMCP databases, including inpatient and outpatient encounters and diagnoses. Because there may be some use of KPMCP facilities without membership, we conducted a sensitivity analysis examining our results among a subcohort of infants who were recorded as members for all 24 months after the infant's birth (see Additional file 1: Tables S1, S2, and S3). The race/ethnicity of participants was based on the self-reported race/ ethnicity of infants' mothers.

\section{Variables and measurements}

To identify only episodes that included a true diagnosis of bronchiolitis while capturing all relevant clinical information associated with a bronchiolitis episode, we classified relevant International Classification of Diseases (ICD-9) codes into two categories. Category A included diagnostic codes specific for bronchiolitis in children younger than two years (ie, 466.1 acute bronchiolitis, $466.1 \mathrm{x}$ acute bronchiolitis organism specified, 466.0 acute bronchitis, 480.0-480.2 pneumonia due to adenovirus, RSV or parainfluenza, 079.0 adenovirus and 079.6 RSV). Category B included diagnostic codes likely to be highly related to bronchiolitis in a child with a current diagnosis of bronchiolitis (i.e., 786.06 tachypnea, 786.07 wheezing, 786.09 other respiratory distress, 780.6$780.60,786.2$ cough, 460 acute nasopharyngitis, 465 acute upper respiratory infections, 465.9 acute upper respiratory infection not otherwise specified, and 487.1 acute upper respiratory infection due to influenza.)

We defined a bronchiolitis episode as a period that included a diagnostic code specific for bronchiolitis from Category A, began either with the first bronchiolitisrelated diagnostic code from Category B occurring within 2 days prior to the first Category A code or with the first Category A code if there was no Category B 


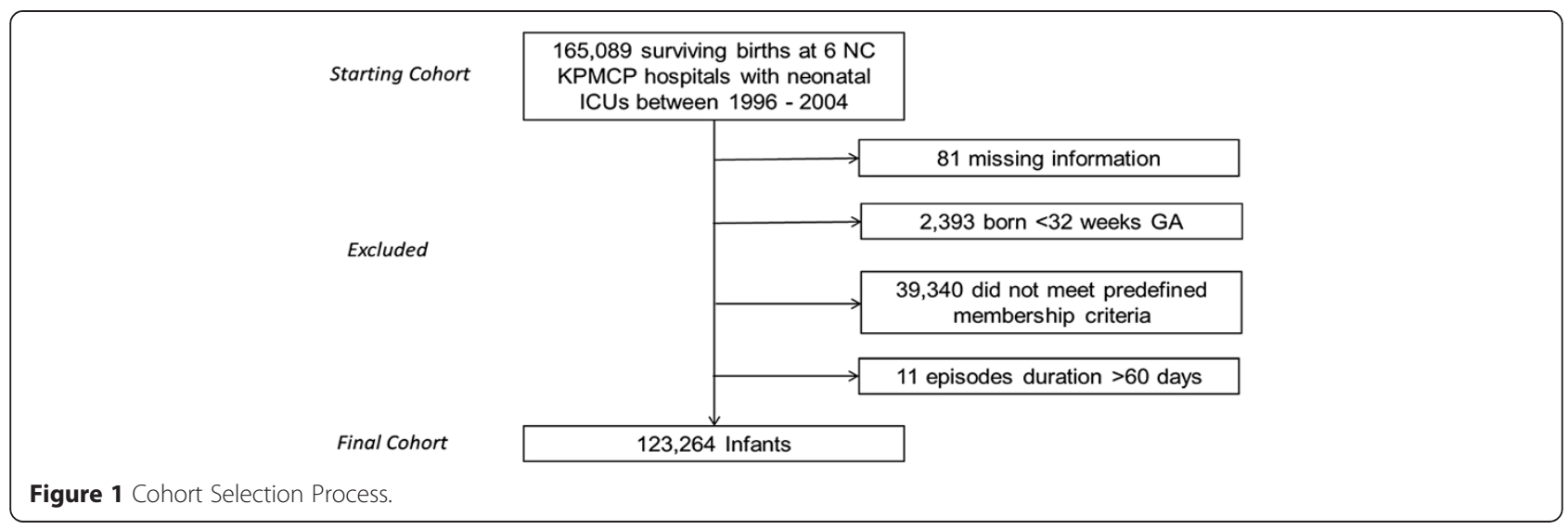

code within 2 days prior to the first Category A code, and ended with a diagnostic code from either Category A or Category B followed by 14 clear days without a bronchiolitis or related diagnosis. An episode of care can include multiple healthcare visits and/or various types of healthcare visits, including hospitalization or outpatient visits with the aforementioned category codes. The duration of an episode of care is defined as the length of time between the first and last visit.

For all infants, we extracted data regarding additional clinical predictors including GA, sex, race/ethnicity, maternal age, birth weight, a diagnosis of BPD or congenital anomaly (ICD-9 codes 425.3x, 425.4x, 425.8x, 745.xx, 746.xx, 747.xx) and the number of siblings present in the home $(\geq 1$ sibling $<5$ years of age). For hospitalization records, we also determined whether the infant required assisted ventilation (ICD-9 codes 93.90, 93.91, and 96.7x).

We classified infants as being small for gestational age (SGA) ( $<5$ th percentile) using birth weight and GA according to the algorithm developed by Brenner et al. [19]. We fitted a smooth cubic spline as a function of total oxygen exposure separately for births occurring at 32-37 and $\geq 37$ weeks GA. Both curves showed a flat effect for total oxygen exposure $<200$ hours and a clear increase at $>200$ hours, suggesting a step function and no interaction between GA and total length of oxygen therapy. For infants treated in the neonatal intensive care unit, we created an oxygen exposure variable: no supplemental oxygen exposure during the neonatal period, no bronchopulmonary dysplasia (BPD); supplemental oxygen exposure of 1 to $<200$ hours during the neonatal period, no BPD; supplemental exposure $\geq 200$ hours during the neonatal period, no BPD; and BPD. We classified infants who did not require intensive care during the neonatal period as having no supplemental oxygen exposure.

Parental asthma history was established by scanning the parent's records for the period 18 months before to 6 months after the infant's birth and determining whether the parent had $\geq 2$ clinical visits 14 days apart with an ICD-9 code (493.xx) for asthma, and/or the parent's electronic record listed asthma on their Significant Problem List.

\section{Statistical analysis}

All statistical analyses used SAS (version 9.1, SAS Institute, Inc., Cary, NC) or Stata (version 9.2, Stata Corp., College Station, TX). After identifying duration of episode, we eliminated episodes lasting $>60$ days from further analysis because it was unlikely that these represented isolated bronchiolitis (see Additional file 1: Table S4). Manual chart review of infants with episodes lasting $>60$ days confirmed that these episodes represented hospitalizations for which bronchiolitis was not the primary diagnosis. Infants remaining after this step constituted the final analysis cohort. We used descriptive statistics to report frequency and duration of bronchiolitis episodes and frequency of hospitalization associated with the episode, by age at the episode, gender, GA, and race/ethnicity. We used a moving average of GA and GA minus 1 week to develop a graph of mean, 10th percentile and 90th percentile for the duration of EOC for each GA. We used multivariable logistic regression to assess the effect of GA, gender, race/ethnicity, family history of asthma, neonatal oxygen use and other clinical predictors on the outcome of whether an infant had a documented bronchiolitis EOC and on the outcome of whether an infant had a documented bronchiolitis EOC lasting $>1$ day. We used White race/ethnicity as the referent category for this multivariate analysis because this was the largest population in our cohort and because previous literature has described disparate risk of bronchiolitis for non-White racial/ethnic groups. We calculated the relative contribution of each predictor using the differences between the log likelihood of the full model and the log likelihood of a model without each of the predictors, and the relative contribution of each predictor was defined as the ratio of its $\log$ 
likelihood difference to the sum of the likelihood differences from all predictors $\times 100$ [20].

\section{Results}

We selected our cohort from 165,089 surviving births during 1996 to 2004 at the six Northern California KPMCP hospitals equipped with neonatal intensive care units. From this original birth population we removed 81 infants with missing information, 2,393 infants born $<32$ weeks GA, 39,340 infants who did not meet predefined membership criteria, and an additional 11 infants with episode duration $>60$ days, resulting in a final cohort of 123,264 infants. The demographics and clinical characteristics of the cohort are presented in Table 1.

Our main analysis focused on bronchiolitis episodes, which included $\geq 1$ medically attended visit for bronchiolitis. Among all infants (123,264), 19,968 infants developed $\geq 1$ episode for bronchiolitis during their first two years of life. Overall, there were 23,748 episodes identified throughout the study period. Among infants who experienced an episode, the mean number of episodes per infant was 1.2 within the first two years of life, so that $16.2 \%$ of infants eventually developed bronchiolits. The overall mean $( \pm \mathrm{SD})$ duration of episodes was $3.2 \pm 4.7$ days. Overall, 2,036 (8.6\%) episodes included a

Table 1 Demographic and clinical characteristics of the main cohort and subgroups

\begin{tabular}{|c|c|c|c|}
\hline & All infants, n (\%) & $\begin{array}{l}\text { Infants with any } \\
\text { bronchiolitis episode } \\
\text { before age } 2 \text { y, n (\%) }\end{array}$ & $\begin{array}{c}\text { Infants with any bronchiolitis } \\
\text { episode before age } 2 \text { y } \\
\text { lasting }>1 \mathrm{~d}, \mathrm{n}(\%)\end{array}$ \\
\hline Sex (Male) & $63,054(51.2)$ & $11,730(58.7)$ & $5,206(60.1)$ \\
\hline \multicolumn{4}{|l|}{ Race $^{a}$} \\
\hline White & $52,609(42.7)$ & $8,305(41.6)$ & $3,754(43.4)$ \\
\hline African-American & $10,374(8.4)$ & $1,866(9.3)$ & $740(8.6)$ \\
\hline Asian & $24,977(20.3)$ & $3,599(18.0)$ & $1,505(17.4)$ \\
\hline Hispanic & $25,619(20.8)$ & $4,704(23.6)$ & $2,047(23.6)$ \\
\hline Other/unknown & $9,685(7.9)$ & $1,494(7.5)$ & $612(7.1)$ \\
\hline \multicolumn{4}{|l|}{ Gestational age, wk } \\
\hline $32-33$ & $1,649(1.3)$ & $406(2.0)$ & $218(2.5)$ \\
\hline $34-36$ & $7,969(6.5)$ & $1,622(8.1)$ & $830(9.6)$ \\
\hline 37 & $7,855(6.4)$ & $1,400(7.0)$ & $632(7.3)$ \\
\hline $38-40$ & $86,708(70.3)$ & $13,701(68.6)$ & $5,834(67.4)$ \\
\hline$\geq 41$ & $19,083(15.5)$ & $2,839(14.2)$ & $1,144(13.2)$ \\
\hline Small for gestational age & $1,763(1.4)$ & $323(1.6)$ & $168(1.9)$ \\
\hline Congenital anomaly present $^{c}$ & $8,646(7.0)$ & $1,642(8.2)$ & $764(8.8)$ \\
\hline \multicolumn{4}{|l|}{ Family history of asthma ${ }^{\mathrm{d}}$} \\
\hline None & $116,763(94.7)$ & $18,618(93.2)$ & $8,011(92.5)$ \\
\hline Father only & $1,824(1.5)$ & $336(1.7)$ & $160(1.9)$ \\
\hline Mother only & $4,561(3.7)$ & $989(5.0)$ & $476(5.5)$ \\
\hline Both parents & $116(0.1)$ & $25(0.1)$ & $11(0.1)$ \\
\hline$\geq 1$ sibling $<5$ y of age in home & $44,235(35.9)$ & $8,405(42.1)$ & $3,801(43.9)$ \\
\hline \multicolumn{4}{|l|}{$\mathrm{O}_{2}$ exposure and $\mathrm{BPD}^{\mathrm{e}}$} \\
\hline $\mathrm{No} \mathrm{O}_{2}$ exposure, no BPD & $118,599(96.2)$ & $18,941(94.9)$ & $8,126(93.9)$ \\
\hline$<200 \mathrm{hrO}$, no BPD & $4,117(3.3)$ & $867(4.3)$ & $427(4.9)$ \\
\hline$\geq 200 \mathrm{hr} \mathrm{O}_{2}$, no BPD & $466(0.4)$ & $138(0.7)$ & $89(1.0)$ \\
\hline $\mathrm{BPD}^{\mathrm{f}}$ & $82(0.1)$ & $22(0.1)$ & $16(0.2)$ \\
\hline
\end{tabular}

BPD = bronchopulmonary dysplasia.

${ }^{a}$ Classification of race/ethnicity in this study was based on the race of infants' mothers, as self-reported to the birth certificate clerk interviewing them at the Kaiser Permanente hospitals described in this study. Race was assessed in this study because of the well-known differences in RSV severity by race.

${ }^{\mathrm{b}}$ Based on the algorithm of Brenner WE, et al. [19].

'See text for list of included International Classification of Diseases codes.

${ }^{d}$ Ascertained by electronic scanning of parental records, which included encounters and diagnoses identified in the Significant Problem List by the parent's physician.

'Oxygen exposure, in hours, during the birth hospitalization. See text for details.

${ }^{f}$ All infants with BPD had $\geq 200$ hours of oxygen exposure during the neonatal period. 
Table 2 Episode frequency and duration and associated hospitalization during the first two years of life

\begin{tabular}{lccccccc}
\hline $\begin{array}{c}\text { Gestational } \\
\text { age, } \mathbf{w k}\end{array}$ & Infants, $\mathbf{n}$ & $\begin{array}{c}\text { Infants with } \\
\text { at least one } \\
\text { EOC, } \mathbf{n}\end{array}$ & $\begin{array}{c}\text { Rate of at least } \\
\text { one episode per } \\
\text { thousand children }\end{array}$ & EOC $^{\mathbf{a}}, \mathbf{n}$ & $\begin{array}{c}\text { Mean episode } \\
\text { duration, } \mathbf{d} \text { (SD) }\end{array}$ & $\begin{array}{c}\text { Episode consisting of ED } \\
\text { visit and hospitalization } \\
\text { and lasting }>\mathbf{1} \mathbf{d}^{\mathbf{b}}, \mathbf{n}(\%)\end{array}$ & $\begin{array}{c}\text { Episode with any } \\
\text { hospitalization, } \mathbf{n} \text { (\%) }\end{array}$ \\
\hline $32-33$ & 1,649 & 406 & 246.2 & 523 & $4.4(5.5)$ & $37(14.2)$ & $101(19.3)$ \\
\hline $34-36$ & 7,969 & 1,622 & 203.5 & 2,003 & $3.9(5.0)$ & $32(7.8)$ & $254(12.7)$ \\
\hline 37 & 7,855 & 1,400 & 178.2 & 1,681 & $3.4(4.6)$ & $44(6.4)$ & $151(9.0)$ \\
\hline $38-40$ & 86,708 & 13,701 & 158.0 & 16,172 & $3.3(4.7)$ & $396(6.3)$ & $1,288(8.0)$ \\
\hline$\geq 41$ & 19,083 & 2,839 & 148.8 & 3,369 & $3.2(4.7)$ & $70(5.6)$ & $242(7.2)$ \\
\hline Total & 123,264 & 19,968 & 162.0 & 23,748 & $3.2(4.7)$ & $619(6.6)$ & $2,036(8.6)$ \\
\hline
\end{tabular}

$\mathrm{ED}=$ emergency department. $\mathrm{EOC}=$ Episode of Care.

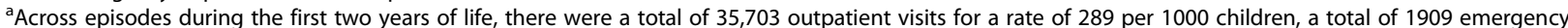
department visits for a rate of 15 per 1000 children and 2169 hospitalizations for a rate of 17.5 per 1000 children.

${ }^{\mathrm{b}}$ Denominator is among episodes $>1$ day for each gestational age cohort.

hospitalization. Of note, 6,351 (26.7\%) included a diagnosis of otitis media.

Among episodes lasting $>1$ day (40\%), the mean duration was $7.0 \pm 5.9$ days; of all episodes, $13.4 \%$ lasted $2-$ 3 days, $11.3 \%$ lasted $4-6$ days, $10.0 \%$ lasted 7-13 days and $5.0 \%$ lasted $\geq 14$ days. The mean number of outpatient visits among episodes lasting $>1$ day was 2.3, while the mean number of emergency department (ED) visits was 0.2 . The majority (85.2\%) of episodes with an ED visit for bronchiolitis had no other healthcare encounters during the episode. However, among children who had an ED visit for bronchiolitis, the mean episode length was 6.3 days and the proportion of infants admitted to the hospital was $35.6 \%(n=626)$.

Preterm birth was associated with both frequency and duration of bronchiolitis episodes (Table 2). The episode rate and corresponding mean duration increased with lower GA. The rate during the first two years of life and mean duration of bronchiolitis EOC were 246 per 1000 infants and $4.4 \pm 5.5$ days, respectively, for infants born at 32-33 weeks and 204 per 1000 infants and $3.9 \pm 5.0$ days, respectively, for infants born at 34-36 weeks GA. In contrast, the rate of episodes among full term infants was lower, 149-178 per 1000 infants. Infants of 32-33 weeks gestational age were also twice as likely as term infants to have a bronchiolitis episode that included an ED visit and a hospitalization $(14.2 \%$ vs. $6.4 \%$ ). Figure 2 shows the increased trend in duration of bronchiolitis episodes in infants with lower GA. We also examined episodes by chronologic age. Overall, 10,380 (43.7\%) episodes occurred in infants $<6$ months of age. For infants $<6$ months of age, the proportion of bronchiolitis episodes including a hospitalization was $11.5 \%$; this proportion was $6 \%$ for those aged 6-24 months.

In our multivariable model, demographic characteristics of male gender and African-American and Hispanic race/ethnicity were associated with increased risk of any episode with odds ratios (OR) of 1.44 (95\% CI, 1.39-
1.48), 1.19 (95\% CI, 1.12-1.25) and 1.20 (95\% CI, 1.151.25), respectively, while adjusting for confounding variables (Table 3). In the same model, Asian race/ethnicity and maternal age $\geq 35$ years were associated with decreased risk of a bronchiolitis episode with OR of 0.91 (95\% CI, 0.87-0.95) and 0.90 (95\% CI, 0.86-0.93), respectively. Gestational age was an important predictor of any bronchiolitis episode, with infants at 32-33 weeks GA having an OR of 1.57 (95\% CI, 1.39-1.77), infants 34-36 weeks GA having an OR of 1.32 (95\% CI, 1.241.40), infants at 37 weeks GA having an OR of 1.15 (95\% CI, 1.08-1.22), and infants $\geq 41$ weeks GA having an OR of 0.95 (95\% CI, 0.90-0.99) for the outcome of any episode when compared with infants 38-40 weeks GA. Other significant risk factors for bronchiolitis episodes included small for GA, presence of congenital anomaly, family history of asthma, at least one sibling $<5$ years of age in the home, and degree of oxygen exposure or having BPD.

The relative contribution of predictors to the overall predictive ability of our model was as follows: gender accounted for $36.0 \%$, while presence of a sibling $<5$ years

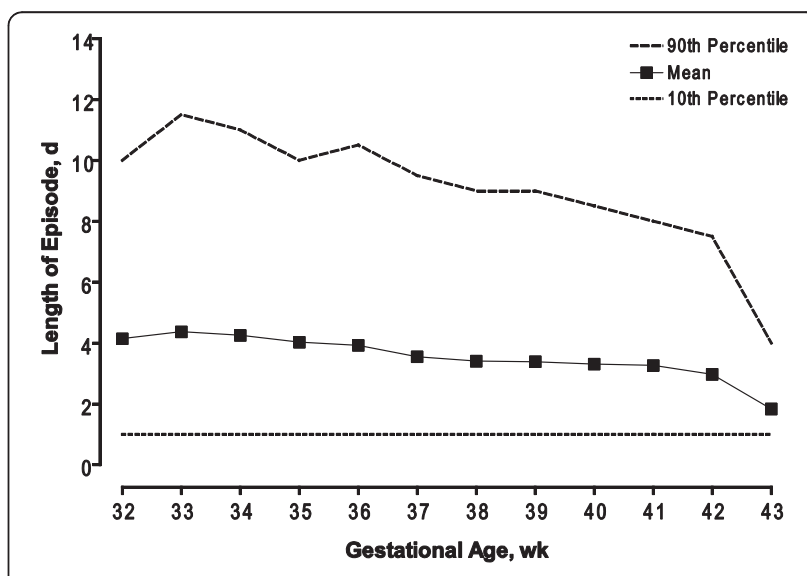

Figure 2 Bronchiolitis episode of care duration as a function of gestational age. 
Table 3 Effect of demographic and clinical predictors on eventual bronchiolitis episodes in multivariate analysis

\begin{tabular}{|c|c|c|}
\hline Clinical and demographic predictors & Beta & Odds Ratio $(95 \% \mathrm{Cl})$ \\
\hline Sex (Male) & 0.36 & $1.44(1.39-1.48)$ \\
\hline \multicolumn{3}{|l|}{ Race $^{a}$} \\
\hline White & 1.00 & reference \\
\hline African-American & 0.17 & $1.19(1.12-1.25)$ \\
\hline Asian & -0.10 & $0.91(0.87-0.95)$ \\
\hline Hispanic & 0.18 & $1.20(1.15-1.25)$ \\
\hline Other/unknown & -0.01 & $0.99(0.93-1.05)$ \\
\hline \multicolumn{3}{|l|}{ Gestational age, wk } \\
\hline $32-33$ & 0.45 & $1.57(1.39-1.77)$ \\
\hline $34-36$ & 0.27 & $1.32(1.24-1.40)$ \\
\hline 37 & 0.14 & $1.15(1.08-1.22)$ \\
\hline $38-40$ & 1.00 & reference \\
\hline$\geq 41$ & -0.06 & $0.95(0.90-0.99)$ \\
\hline Small for gestational age ${ }^{b}$ & 0.16 & $1.17(1.03-1.32)$ \\
\hline Congenital anomaly present $^{c}$ & 0.13 & $1.14(1.08-1.21)$ \\
\hline \multicolumn{3}{|l|}{ Family history of asthma ${ }^{d}$} \\
\hline None & 1.00 & \\
\hline Father only & 0.16 & $1.17(1.04-1.32)$ \\
\hline Mother only & 0.37 & $1.45(1.35-1.56)$ \\
\hline Both parents & 0.38 & $1.46(0.94-2.29)$ \\
\hline$\geq 1$ sibling $<5$ y of age in home & 0.33 & $1.39(1.35-1.44)$ \\
\hline \multicolumn{3}{|l|}{$\mathrm{O}_{2}$ exposure and $\mathrm{BPD}^{\mathrm{e}}$} \\
\hline $\mathrm{No} \mathrm{O}_{2}$ exposure, no BPD & 1.00 & reference \\
\hline$<200 \mathrm{hr} \mathrm{O}_{2}$, no BPD & 0.17 & $1.19(1.10-1.29)$ \\
\hline$\geq 200 \mathrm{hr} \mathrm{O}_{2}$, no BPD & 0.58 & $1.79(1.46-2.20)$ \\
\hline BPD $^{f}$ & 0.28 & $1.33(0.80-2.19)$ \\
\hline \multicolumn{3}{|l|}{ Maternal age, y } \\
\hline$<18$ & 0.09 & $1.10(0.96-1.25)$ \\
\hline $18-34$ & 1.00 & reference \\
\hline$\geq 35$ & -0.11 & $0.90(0.86-0.93)$ \\
\hline
\end{tabular}

$\mathrm{BPD}=$ bronchopulmonary dysplasia.

aClassification of race/ethnicity in this study was based on the race of infants' mothers, as self-reported to the birth certificate clerk interviewing them at the Kaiser Permanente hospitals described in this study. Race was assessed in this study because of the well-known differences in RSV severity by race.

${ }^{\mathrm{b}}$ Based on the algorithm of Brenner WE, et al. [19].

'See text for list of included International Classification of Diseases codes. ${ }^{\mathrm{d}}$ Ascertained by electronic scanning of parental records, which included encounters and diagnoses identified in the Significant Problem List by the parent's physician.

'Oxygen exposure, in hours, during the birth hospitalization. See text for details.

${ }^{f}$ All infants with BPD had $\geq 200$ hours of oxygen exposure during the neonatal period.

of age accounted for $28.5 \%$, race/ethnicity accounted for $11.3 \%$, and GA accounted for $10.1 \%$. The contribution of other predictors was $<7 \%$ each (see Additional file 1 : Table S5). In multivariable analyses, predictors for having any episode were similar to predictors for having an episode lasting $>1$ day (Table 4; see also Additional file 1: Table S5). Results for both multivariate models were similar for the sensitivity analysis cohort (see Additional file 1: Table S2 and Additional file 1: Table S3).

\section{Discussion}

Our study shows that bronchiolitis care episodes were frequent during the first two years of life in this study cohort and that, consistent with prior research, most occurred outside the hospital setting. In our large cohort, the duration of bronchiolitis episodes ranged from 1 to 27 days. Among episodes with duration $>1$ day, the mean duration was $7.0 \pm 5.9$ days, potentially indicating significant morbidity for this subset of infants. Preterm infants compared to full-term infants had higher overall bronchiolitis episode rates as well as higher rates of episodes with an ED and/or inpatient hospital admission during the first two years of life, which might reflect both an intrinsically higher risk of morbidity in this population and/or an increased level of parent and provider concern regarding their medical needs. In addition to prematurity, male gender, neonatal oxygen use, family history of asthma, and African-American or Hispanic race/ethnicity were associated with a higher frequency of at least one episode and/or a higher frequency of an episode $>1$ day.

Consistent with previous studies, the overall burden of disease in this population was relatively high, with $16.2 \%$ of children in this study having a documented episode of bronchiolitis before age two. An episode of care approach in characterizing the utilization patterns of infants and children with bronchiolitis may be useful for health services researchers, physicians, and parents in understanding a broader healthcare burden of disease. Analyzing episodes of care may be more informative than reporting the rates of discrete bronchiolitis-related medical encounters.

Our study has several important limitations. First, not all infants with bronchiolitis seek medical care. This study reports frequency and associated characteristics of bronchiolitis that led to a clinical encounter, but is not a prospective study of the entire burden of illness from bronchiolitis that includes patient reports of symptoms [21]. Nevertheless, the study design provided a good approximation of the total health care utilization associated with bronchiolitis. Second, we ascertained bronchiolitis diagnoses using ICD-9 codes. To be considered an episode, a subject needed to have $\geq 1$ diagnostic code specific for bronchiolitis or viral pneumonia attributable to pathogens typically causing bronchiolitis (Category A). Because bronchiolitis is a clinical diagnosis, it is possible that some cases of bronchiolitis were instead coded as "fever," "wheeze," or "lower respiratory infection."However, it is also possible that some pneumonia attributed to pathogens typically causing bronchiolitis was not in fact bronchiolitis, and that some episodes of asthma, viral pneumonia or upper respiratory infection were coded as 
Table 4 Effect of demographic and clinical predictors on eventual bronchiolitis episodes $>1$ day in multivariate analysis

\begin{tabular}{|c|c|c|}
\hline Clinical and demographic predictors & Beta & Odds Ratio $(95 \% \mathrm{Cl})$ \\
\hline Sex (Male) & 0.38 & $1.47(1.40-1.54)$ \\
\hline \multicolumn{3}{|l|}{ Race $^{a}$} \\
\hline White & 1.00 & reference \\
\hline African-American & 0.01 & $1.01(0.93-1.10)$ \\
\hline Asian & -0.17 & $0.85(0.79-0.90)$ \\
\hline Hispanic & 0.13 & $1.13(1.07-1.20)$ \\
\hline Other/unknown & -0.11 & $0.89(0.82-0.98)$ \\
\hline \multicolumn{3}{|l|}{ Gestational age, wk } \\
\hline $32-33$ & 0.58 & $1.79(1.53-2.09)$ \\
\hline $34-36$ & 0.43 & $1.53(1.41-1.66)$ \\
\hline 37 & 0.18 & $1.20(1.10-1.31)$ \\
\hline $38-40$ & 1.00 & reference \\
\hline$\geq 41$ & -0.11 & $0.90(0.84-0.96)$ \\
\hline Small for gestational age $\mathrm{b}^{\mathrm{b}}$ & 0.32 & $1.37(1.16-1.61)$ \\
\hline Congenital anomaly present ${ }^{c}$ & 0.15 & $1.17(1.08-1.27)$ \\
\hline \multicolumn{3}{|l|}{ Family history of asthma ${ }^{d}$} \\
\hline None & 1.00 & reference \\
\hline Father only & 0.24 & $1.27(1.08-1.50)$ \\
\hline Mother only & 0.45 & $1.56(1.41-1.72)$ \\
\hline$\geq 1$ sibling $<5$ y of age in home & 0.37 & $1.45(1.39-1.52)$ \\
\hline \multicolumn{3}{|l|}{$\mathrm{O}_{2}$ exposure and $\mathrm{BPD}^{\mathrm{e}}$} \\
\hline $\mathrm{No} \mathrm{O}_{2}$ exposure, no BPD & 1.00 & reference \\
\hline$<200 \mathrm{hr}{ }_{2}$, no BPD & 0.22 & $1.25(1.12-1.39)$ \\
\hline$\geq 200 \mathrm{hr} \mathrm{O}_{2}$, no BPD & 0.88 & $2.41(1.89-3.07)$ \\
\hline BPD $^{f}$ & 0.69 & $1.99(1.13-3.49)$ \\
\hline \multicolumn{3}{|l|}{ Maternal age, y } \\
\hline$<18$ & 0.03 & $1.03(0.85-1.26)$ \\
\hline $18-34$ & 1.00 & reference \\
\hline$\geq 35$ & -0.09 & $0.91(0.86-0.96)$ \\
\hline
\end{tabular}

$\mathrm{BPD}=$ bronchopulmonary dysplasia.

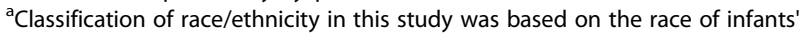
mothers, as self-reported to the birth certificate clerk interviewing them at the Kaiser Permanente hospitals described in this study. Race was assessed in this study because of the well-known differences in RSV severity by race.

${ }^{b}$ Based on the algorithm of Brenner WE, et al. [19].

'See text for list of included International Classification of Diseases codes.

${ }^{d}$ Ascertained by electronic scanning of parental records, which included encounters and diagnoses identified in the Significant Problem List by the parent's physician.

e Oxygen exposure, in hours, during the birth hospitalization. See text for details.

${ }^{f}$ All infants with BPD had $\geq 200$ hours of oxygen exposure during the neonatal period.

"bronchiolitis." Therefore, it is likely that the total error from miscoding of bronchiolitis is relatively small. Third, we estimated membership based on a combination of membership and use status in each cohort month. Because infants with a higher level of use might tend to have higher frequency of seeking medical care, it is possible that this might bias the results towards higher estimates of bronchiolitis episodes. Since the sensitivity analysis including only infants with 24 months of documented membership did not show much difference from the primary analysis, the effect of membership status is likely to be minimal. Fourth, there were no reliable data on smoking for this analysis. Because smoking in the home is a major predictor of bronchiolitis episodes and because maternal smoking is associated with preterm birth and with race/ethnicity, it is possible that smoking might confound the relationship between GA and incidence of bronchiolitis. However, in our previous work we have found that the overall rate of smoking among mothers delivering at KPMCP is 4\%-8\% [22]. Therefore, we do not believe this has had a large impact on our results. Fifth, because rates of episodes and episodes with hospitalization might be impacted by participation in an integrated healthcare delivery system, it is possible that our results may not be generalizable to other healthcare systems. However, because the KPMCP provides medical care to a large portion of infants born in Northern California, our data represent an important estimate of overall care episodes in this population. Sixth, our data on race/ethnicity include only the categories White, African-American, Asian, Hispanic and other, and we do not have access to more detailed data. Therefore, we cannot report how more specific subgroups of race/ethnicity might alter bronchiolitis risk.

Despite these limitations, our research design offers the unique opportunity to study an entire birth cohort using linked data with relatively little loss to follow-up and to report the health care utilization related to bronchiolitis in this prospective cohort. Thus, we are able to provide a comprehensive description of burden of disease in this population, including frequency and severity of illness. In addition, the large sample size of our cohort has allowed us to identify many predictors associated with the disease outcome, which include gestational age, race/ethnicity, maternal age, small for GA, presence of congenital anomaly, family history of asthma, at least one sibling $<5$ years of age in the home, and degree of oxygen exposure or having BPD.

\section{Conclusions}

In this cohort, $16.2 \%$ of infants had one or more episodes of bronchiolitis in the first two years after birth, with episodes lasting from 1 to 27 days. Preterm infants were more likely to have a bronchiolitis episode compared to term infants and were more likely than term infants to have longer periods of healthcare utilization, perhaps reflecting both the medical needs of this particular high-risk population group and the vulnerability perceived by providers and parents. Using an episode of care approach to study the epidemiology of bronchiolitis 
may provide additional information about burden of disease for both preterm and term infants.

\section{Additional file}

\section{Additional file1 Online-Only Material Sensitivity Analyses}

\section{Competing interests}

Dr. Anthony Masaquel is an employee of Medlmmune, LLC, the company which funded the study reported here.

\section{Acknowledgments}

This project was conducted and funded under a contract with Medlmmune, LLC. Under the terms of this contract, Dr. Escobar was the principal investigator for the overall project and had full access to all data in the study and supervised data collection and data cleaning. For this paper, Dr. Flaherman was the principal investigator, and, in conjunction with Dr. Escobar and Dr. Kipnis, the project statistician, supervised all project analyses. Medlmmune was involved with the design of the study, interpretation of the data, and review of the manuscript. All authors agreed on the final text and conclusions of the manuscript, which were also reviewed by Dr. Joseph V. Selby, Director of the Kaiser Permanente Division of Research and Dr. Parthiv J. Mahadevia, Senior Director, Health Outcomes and Pharmacoeconomics, Medlmmune, LLC, Assistance formatting the manuscript for submission was provided by Complete Healthcare Communications, Inc. (Chadds Ford, PA) and funded by Medlmmune, LLC.

The study was sponsored by Medlmmune, LLC

\section{Author details}

'Department of Pediatrics, University of California, 3333 California St, Box 0503, San Francisco, CA 94143-0503, USA. ²Division of Research, Kaiser Permanente Medical Care Program, Systems Research Initiative and Perinatal Research Unit, 2000 Broadway, 2nd floor, Oakland, CA 94612, USA. ${ }^{3}$ Kaiser Foundation Health Plan, Inc, Management Information and Analysis, 1950 Franklin St, 17th floor, Oakland, CA 94612, USA. ${ }^{4}$ Medlmmune, LLC, One Medlmmune Way, Gaithersburg, MD 20878, USA. ${ }^{5}$ Department of Inpatient Pediatrics, Kaiser Permanente Medical Center, 1425 S. Main St, Walnut Creek, CA 94596, USA.

\section{Authors' contributions}

VF participated in the conception and design of this study, participated in the analysis of the data, drafted the article, and has given final approval for the version to be published. AR participated in the conception and design of this study, participated in the analysis of the data, revised the manuscript critically for important intellectual content, and has given final approval for the version to be published. SXL participated in the conception and design of this study, analyzed the data, revised the manuscript critically for important intellectual content, and has given final approval for the version to be published. PK participated in data analysis, revised the manuscript critically for important intellectual content, and has given final approval for the version to be published. AM conceived and designed this study, revised the manuscript critically for important intellectual content, and has given final approval for the version to be published. GE conceived and designed this study, participated in the analysis of the data, revised the manuscript critically for important intellectual content, and has given final approval for the version to be published. All authors read and approved the final manuscript.

Received: 14 September 2011 Accepted: 8 June 2012

Published: 8 June 2012

\section{References}

1. Subcommittee on Diagnosis and Management of Bronchiolitis: Diagnosis and management of bronchiolitis. Pediatrics 2006, 118(4):1774-1793.

2. Mansbach JM, Pelletier AJ, Camargo CA Jr: US outpatient office visits for bronchiolitis, 1993-2004. Ambul Pediatr 2007, 7(4):304-307.

3. Yorita KL, Holman RC, Sejvar JJ, Steiner CA, Schonberger LB: Infectious disease hospitalizations among infants in the United States. Pediatrics 2008, 121(2):244-252.
4. Pelletier AJ, Mansbach JM, Camargo CA Jr: Direct medical costs of bronchiolitis hospitalizations in the United States. Pediatrics 2006, 118 (6):2418-2423

5. Carroll KN, Gebretsadik T, Griffin MR, et al: Increasing burden and risk factors for bronchiolitis-related medical visits in infants enrolled in a state health care insurance plan. Pediatrics 2008, 122(1):58-64.

6. Leader $\mathrm{S}$, Kohlhase $\mathrm{K}$ : Recent trends in severe respiratory syncytial virus (RSV) among US infants, 1997 to 2000. J Pediatr 2003, 143(5 Suppl): S127-S132.

7. Rosychuk RJ, Klassen TP, Voaklander DC, Senthilselvan A, Rowe BH: Presentations of infants to emergency departments in Alberta, Canada, for bronchiolitis: a large population-based study. Pediatr Emerg Care 2011, 27(3):189-195.

8. Pezzotti P, Mantovani J, Benincori N, Mucchino E, Di Lallo D: Incidence and risk factors of hospitalization for bronchiolitis in preterm children: a retrospective longitudinal study in Italy. BMC Pediatr 2009, 9:56.

9. Thorburn K: Pre-existing disease is associated with a significantly higher risk of death in severe respiratory syncytial virus infection. Arch Dis Child 2009, 94(2):99-103.

10. Koehoorn M, Karr CJ, Demers PA, Lencar C, Tamburic L, Brauer M: Descriptive epidemiological features of bronchiolitis in a populationbased cohort. Pediatrics 2008, 122(6):1196-1203.

11. Shay DK, Holman RC, Newman RD, Liu LL, Stout JW, Anderson LJ: Bronchiolitis-associated hospitalizations among US children, 1980-1996. JAMA 1999, 282(15):1440-1446.

12. Hall CB, Weinberg GA, Iwane MK, et al: The burden of respiratory syncytial virus infection in young children. New Engl J Med 2009, 360(6):588-598.

13. Hornbrook MC, Hurtado AV, Johnson RE: Health care episodes: definition, measurement and use. Med Care Rev Fall 1985, 42(2):163-218.

14. Hall CB: Diagnosis and testing in bronchiolitis: a systematic review. J Pediatr 2004, 145(3):417-418.

15. Rosen AK: Windows of observation. In Risk Adjustment for Measuring Health Care Outcomes. Edited by lezzoni L. Chicago: Health Administration Press; 2003:71-82.

16. Lohr KN, Brook RH: Quality of care in episodes of respiratory illness among Medicaid patients in New Mexico. Ann Intern Med 1980, 92(1):99_ 106.

17. Boyce TG, Weaver AL, St Sauver JL, Stancl JK, Woodward-Lee AE: Incidence of bronchiolitis-associated hospitalization among children in Olmsted County, Minnesota. Mayo Clin Proc 2004, 79(6):832-833.

18. Hamilton BE, Sutton PD, Mathews TJ, Martin JA, Ventura SJ: The effect of Hurricane Katrina: births in the U.S. Gulf Coast region, before and after the storm. Natl Vital Stat Rep 2009, 58(2):1-28. 32.

19. Brenner WE, Edelman DA, Hendricks CH: A standard of fetal growth for the United States of America. Am J Obstetric and Gynecol 1976, 126(5):555-564.

20. Render ML, Kim HM, Welsh DE, et al: Automated intensive care unit risk adjustment: results from a National Veterans Affairs study. Crit Care Med 2003, 31(6):1638-1646.

21. Petruzella FD, Gorelick MH: Duration of illness in infants with bronchiolitis evaluated in the emergency department. Pediatrics 2010, 126(2):285-290.

22. Escobar GJ, Ragins A, Li SX, Prager L, Masaquel AS, Kipnis P: Recurrent wheezing in the third year of life among children born at $>32$ weeks gestation: relationship to laboratory-confirmed, medically attended infection with respiratory syncytial virus during the first year of life. Arc Pediatr Adolesc Med. In press.

doi:10.1186/1472-6963-12-144

Cite this article as: Flaherman et al.: Frequency, duration and predictors of bronchiolitis episodes of care among infants $\geq 32$ weeks gestation in a large integrated healthcare system: a retrospective cohort study. BMC Health Services Research 2012 12:144. 